\title{
A NOTE ON A MULTIPLIER OF THE NORLUND MEANS AND CONVEX MAPS OF THE UNIT DISC
}

\author{
ZIAD S. ALI
}

Received 27 January 2005 and in revised form 3 June 2005

Important and interesting results which are already known such as those by Pólya and Schoenberg in (1958), Başgöze et al. in (1970), and Ali in (1973), and which are related to convex maps of the unit disc are presented in this note by a more general, unified, and different method. The method considers the product of a nontrivial multiplier with the Norlund means, and shows how the known results indicated above are reproduced again as special cases.

\section{Introduction}

Let $\sum_{k=0}^{\infty} u_{k}$ be a given series and let $\left\{S_{n}\right\}_{0}^{\infty}$ denote the sequence of its partial sums. Let $\left\{q_{n}\right\}$ be a sequence of real numbers with $q_{j}>0$ for some $j=0,1,2, \ldots$, and $q_{n} \geq 0$ for all $n=0,1,2, \ldots, n \neq j$, and let $Q_{n}=\sum_{j=0}^{n} q_{j}$.

By Hardy [6], the sequence-to-sequence transformation defined by

$$
T_{n}=\frac{1}{Q_{n}} \sum_{j=0}^{n} q_{n-j} S_{j}
$$

is called the Norlund means of $\left\{S_{n}\right\}_{0}^{\infty}$, and is denoted by $\left(N, q_{n}\right)$. The $\left(N, q_{n}\right)$ is regular if and only if $q_{n}=o\left(Q_{n}\right)$ as $n \rightarrow \infty$.

By Duren [4], a domain $E$ of the complex plane is said to be convex if and only if the line segment joining any two points of $E$ lies entirely in $E$. A function $f$ which is analytic, univalent in the unit disc $D=\{z: z<1\}$, and is normalized by $f(0)=f^{\prime}(0)-1=0$ is said to belong to the class $K$ if and only if it is a conformal mapping of the unit disc $D=$ $\{z: z<1\}$ onto a convex domain. Equivalently, $f \in K$ if and only if $\operatorname{Re} \cdot\left(1+z f^{\prime \prime}(z) /\right.$ $\left.f^{\prime}(z)\right)>0$.

Additional information on convexity are given in [8] by Pólya and Schoenberg, in [3] by Başgöze et al., and in [1] by Ali. Clearly with the normalized conditions imposed on $f$, it is easily seen that $a_{0}=0$ and $a_{1}=1$. From now on when talking about $f$, we imply that $f$ is an element of the class $K$ if this fact is already not indicated. 
3352 Multiplier of Norlund means and convex maps of unit disc

Let $g$ and $h$ be analytic in $D$, by Nehari [7], $g$ is said to be subordinate to $h$ written as

$$
g \prec h \quad \text { or } \quad g(z) \prec h(z), \quad z \in D,
$$

if $g(0)=h(0)$, and $g(D)$ subset of $h(D)$.

\section{Means connected with power series}

Let

$$
\begin{aligned}
& S_{n}(z, f)=\sum_{j=1}^{n} a_{j} z^{j} \text { be the sequence of partial sums of } f, \\
& \sigma_{n}(z, f)=\frac{1}{n} \sum_{j=1}^{n} S_{j}(z, f) \text { the Cesaro means or the }(C, 1) \text { means of } f \\
& T_{n}(z, f)=\frac{1}{Q_{n}} \sum_{j=1}^{n} q_{n-j} S_{j}(z, f) \text { the Norlund means of } f, \\
& V_{n}(z, f)=\frac{1}{\left(\begin{array}{c}
2 n \\
n
\end{array}\right)} \sum_{j=1}^{n}\left(\begin{array}{c}
2 n \\
n+j
\end{array}\right) a_{j} z^{j} \text { the de la Vallee Poussin means of } f .
\end{aligned}
$$

\section{Known results}

In [8], Pólya and Schoenberg proved the following theorem.

Theorem 3.1. For $f(z) \in K$, it is necessary and sufficient that $V_{n}(z, f) \in K$ for $n=1,2, \ldots$

In [3], Başgöze et al. proved the following theorem.

Theorem 3.2. (i) Suppose that the values taken by $f(z)$ for $z$ in $D$ lie in a convex domain $D_{w}$; then the values taken by $\sigma_{n}(z, f)$ also lie in $D_{w}$ for all $n$ and all $z$ in $D$.

(ii) Conversely, suppose that the values taken by $\sigma_{n}(z, f)$ lie in a convex domain $D_{w}$; then the values taken by $f(z)$ lie in $D_{w}$ for all $z$ in $D$.

In [1], Ali proved the following theorem.

Theorem 3.3. (i) Let $\left(N, q_{n}\right)$ be a regular Norlund transformation such that $\left\{q_{n}\right\}_{0}^{\infty}$ is a nondecreasing sequence of positive numbers. Suppose that the values taken by $f(z)$, for $z$ in $D$, lie in a convex domain $D_{w}$, then the values taken by $T_{n}(z, f)$ also lie in $D_{w}$ for all $n$ and all $z$ in $D$.

(ii) Conversely, suppose that the values taken by $T_{n}(z, f)$ lie in a convex domain $D_{w}$; then the values taken by $f(z)$ lie in $D_{w}$ for all $z$ in $D$.

In [2], Ali has the following theorem.

Theorem 3.4. (i) Let $f \in K$.

(ii) Let

$$
\begin{gathered}
Q_{n}=\sum_{j=0}^{n} q_{j}, \quad Q_{j}=\left(\begin{array}{c}
2 n \\
j
\end{array}\right) q_{0}, \\
\omega(n)=\frac{-2}{Q_{n}} \sum_{j=1}^{n}(-1)^{j} Q_{n-j},
\end{gathered}
$$


then

$$
\frac{1}{\omega(n)} T_{n}(z, f) \prec f
$$

\section{The main theorem}

In this note, we prove a theorem concerning the following product:

$$
\frac{1}{\omega(n)} T_{n}(z, f)
$$

where

$$
\frac{1}{\omega(n)}=\frac{1}{\left(-2 / Q_{n}\right) \sum_{j=1}^{n}(-1)^{j} Q_{n-j}}, \quad T_{n}(z, f)=\frac{1}{Q_{n}} \sum_{j=1}^{n} q_{n-j} S_{j}(z, f) .
$$

We will actually show that under certain assumptions that the product

$$
\frac{1}{\omega(n)} T_{n}(z, f)
$$

equals to the de la Vallee Pouissin means of $f$ at one time, furthermore equals to the Cesaro means of $f$ at a second time, and equals to the Norlund sums of $f$ at the third time.

The theorem is an outcome of Theorem 3.4 by Ali in [2], and is general to cover the important, and interesting results indicated above by Pólya and Schoenberg [8], another by Başgöze et al. [3], and third by Ali in [1].

In this note, we prove the following theorem.

Theorem 4.1. (i) Let $f \in K$.

(ii) Let

$$
\begin{aligned}
Q_{j}^{n}=\sum_{r=0}^{j} q_{r}^{n} & =\sum_{r=0}^{j} \frac{(2 n-2 r+1)}{(2 n-r+1)}\left(\begin{array}{c}
2 n \\
r
\end{array}\right) q_{0}, \\
\omega(n) & =\frac{-2}{Q_{n}^{n}} \sum_{j=1}^{n}(-1)^{j} Q_{n-j}^{n},
\end{aligned}
$$

then

$$
\frac{1}{\omega(n)} T_{n}(z, f) \in K \quad \text { iff } f \in K
$$

Remark 4.2. Without loss of generality, and in order to be more clear, the notation has been slightly modified. For example, talking about $Q_{n-j}$ before is the same as talking about $Q_{n-j}^{n}$ now, and $q_{n-j}$ before is the same as $q_{n-j}^{n}$ now, similarly $Q_{n}$ before is the same as $Q_{n}^{n}$ now. 
3354 Multiplier of Norlund means and convex maps of unit disc

Proof of Theorem 4.1. We first note by Graham et al. [5] that

$$
Q_{j}^{n}=\sum_{r=0}^{j} q_{r}^{n}=\sum_{r=0}^{j} \frac{(2 n-2 r+1)}{(2 n-r+1)}\left(\begin{array}{c}
2 n \\
r
\end{array}\right) q_{0}=\left(\begin{array}{c}
2 n \\
j
\end{array}\right) q_{0},
$$

furthermore

$$
\begin{aligned}
\frac{1}{\omega(n)} T_{n}(z, f) & =\frac{1}{\left(-2 / Q_{n}^{n}\right) \sum_{j=1}^{n}(-1)^{j} Q_{n-j}^{n}} \frac{1}{Q_{n}^{n}} \sum_{j=1}^{n} q_{n-j}^{n} S_{j}(z, f), \\
\frac{1}{\omega(n)} T_{n}(z, f) & =\frac{1}{-2 \sum_{j=1}^{n}(-1)^{j} Q_{n-j}^{n}} \sum_{j=1}^{n} Q_{n-j}^{n} a_{j} z^{j} .
\end{aligned}
$$

The next step is to note that

$$
-2 \sum_{j=1}^{n}(-1)^{j} Q_{n-j}^{n}=-\left(\sum_{j=1}^{n}(-1)^{j} Q_{n-j}^{n}+\sum_{j=1}^{n}(-1)^{j} Q_{n+j}^{n}\right) .
$$

Now using the definition of $Q_{j}^{n}$, we can easily see the following.

For $n$ odd, we have

$$
-2 \sum_{j=1}^{n}(-1)^{j} Q_{n-j}^{n}=\sum_{j=0}^{2 n}(-1)^{j}\left(\begin{array}{c}
2 n \\
j
\end{array}\right)+\left(\begin{array}{c}
2 n \\
n
\end{array}\right)
$$

and, for $n$ even, we have

$$
-2 \sum_{j=1}^{n}(-1)^{j} Q_{n-j}^{n}=-\sum_{j=0}^{2 n}(-1)^{j}\left(\begin{array}{c}
2 n \\
j
\end{array}\right)+\left(\begin{array}{c}
2 n \\
n
\end{array}\right) .
$$

Now since

$$
\sum_{j=0}^{2 n}(-1)^{j}\left(\begin{array}{c}
2 n \\
j
\end{array}\right)=0
$$

it follows that

$$
\frac{1}{\omega(n)} T_{n}(z, f)=\frac{1}{\left(\begin{array}{c}
2 n \\
n
\end{array}\right)} \sum_{j=1}^{n}\left(\begin{array}{c}
2 n \\
n+j
\end{array}\right) a_{j} z^{j}=V_{n}(z, f)
$$

which are the de la Vallee Poussin means of $f$, and the theorem follows by Pólya and Schoenberg [8]. 
Corollary 4.3. (i) Let $f \in K$.

(ii) Let

$$
Q_{n}^{n}=\sum_{r=0}^{n} q_{r}^{n}=n+1,
$$

and let

$$
\omega(n)= \begin{cases}\frac{-2}{Q_{n}^{n}} \sum_{j=1}^{n}(-1)^{j} Q_{n-j}^{n}-1, & n \text { is odd }, \\ \frac{-2}{Q_{n}^{n}} \sum_{j=1}^{n}(-1)^{j} Q_{n-j}^{n}, & n \text { is even, }\end{cases}
$$

then

$$
\frac{1}{\omega(n)} T_{n}(z, f) \in K \quad \text { iff } f \in K .
$$

Proof of Corollary 4.3. Clearly $Q_{n-j}^{n}=n-j+1$, and for $n=2 k+1$, we have

$$
\begin{aligned}
-2 \sum_{j=1}^{n}(-1)^{j}(n-j+1)-1 & =-2 \sum_{j=1}^{2 k+1}(-1)^{j}(2 k+2-j)-1 \\
& =-2(-(2 k+1)+k)-1 \\
& =2 k+1=n .
\end{aligned}
$$

Similarly, when considering the case where $n=2 k$ to obtain the following:

$$
n= \begin{cases}-2 \sum_{j=1}^{n}(-1)^{j}(n-j+1)-1, & n \text { is odd, } \\ -2 \sum_{j=1}^{n}(-1)^{j}(n-j+1), & n \text { is even, }\end{cases}
$$

accordingly for any $n$, we have $(1 / \omega(n)) T_{n}(z, f)=(1 / n) \sum_{j=1}^{n} S_{k}(z, f)=\sigma_{n}(z, f)$, which are the Cesaro means of $f$, and the result follows by Başgöze et al. [3].

Corollary 4.4. (i) Let $f \in K$.

(ii) Let $T_{n}(z, f)$ be a Norlund-type transformation defined by a nondecreasing sequence $\left\{q_{n}\right\}_{0}^{\infty}$ of positive real numbers such that $\sum_{f \in \text { odd }} q_{j}^{n}=\sum_{j \in \text { even }} q_{j}^{n}$, where $j$ is a nonnegative integer, then

$$
\frac{1}{\omega(n)} T_{n}(z, f) \in K \quad \text { iff } f \in K
$$


3356 Multiplier of Norlund means and convex maps of unit disc

Proof of Corollary 4.4. For $n=2 k+1$, we have

$$
\begin{aligned}
-2 \sum_{j=1}^{n}(-1)^{j} Q_{n-j}^{n} & =-2 \sum_{j=1}^{2 k+1}(-1)^{j} Q_{2 k+1-j}^{2 k+1} \\
& =2 \sum_{j \in \text { even }}^{2 k-2}\left(Q_{2 k-j}^{2 k+1}-Q_{2 k-j-1}^{2 k+1}\right)+Q_{0}^{2 k+1}, \quad j=0,2, \ldots, \\
& =2 \sum_{j=0}^{2 k} q_{2 k-2 j}^{2 k+1}=2 \sum_{j \in \text { even }}^{n} q_{j}^{n}, \quad j=0,2, \ldots
\end{aligned}
$$

Similarly when $n=2 k$, we get

$$
\begin{aligned}
-2 \sum_{j=1}^{n}(-1)^{j} Q_{n-j}^{n} & =-2 \sum_{j=1}^{2 k}(-1)^{j} Q_{2 k-j}^{2 k} \\
& =2 \sum_{j \in \text { odd }}^{2 k-1}\left(Q_{2 k-j}^{2 k}-Q_{2 k-j-1}^{2 k}\right), \quad j=1,3, \ldots, \\
& =2 \sum_{j \in \text { odd }}^{2 k-1} q_{2 k-j}^{2 k}=2 \sum_{j \in \text { odd }}^{n} q_{j}^{n}, \quad j=1,3, \ldots
\end{aligned}
$$

Hence for $j$ nonnegative integer, we have

$$
\omega(n)=\frac{-2}{Q_{n}^{n}} \sum_{j=1}^{n}(-1)^{j} Q_{n-j}^{n}=\frac{1}{Q_{n}^{n}}\left(\sum_{j \in \text { odd }}^{n} q_{j}^{n}+\sum_{j \in \text { even }}^{n} q_{j}^{n}\right)=1 .
$$

Accordingly, the result follows by Ali [1].

\section{Acknowledgment}

I am thankful to both the referee and the Editor of the journal.

\section{References}

[1] Z. S. Ali, Convexity of Nörlund and progressive means, Indian J. Math. 15 (1973), no. 2, 133136.

[2] - Subordinating factors of a Norlund transformation, Abstracts Amer. Math. Soc. 9 (1988), no. 56, 219.

[3] T. Başgöze, J. L. Frank, and F. R. Keogh, On convex univalent functions, Canad. J. Math. 22 (1970), 123-127.

[4] P. L. Duren, Univalent Functions, Fundamental Principles of Mathematical Sciences, vol. 259, Springer, New York, 1983.

[5] R. L. Graham, D. E. Knuth, and O. Patashnik, Concrete Mathematics: A Foundation for Computer Science, 2nd ed., Addison-Wesley, New York, 1998.

[6] G. H. Hardy, Divergent Series, Clarendon Press, Oxford University Press, New York, 1949.

[7] Z. Nehari, Conformal Mapping, McGraw-Hill, New York, 1952. 
[8] G. Pólya and I. J. Schoenberg, Remarks on de la Vallée Poussin means and convex conformal maps of the circle, Pacific J. Math. 8 (1958), no. 2, 295-334.

Ziad S. Ali: American College of Switzerland, 1854 Leysin, Switzerland E-mail address: alioppp@yahoo.com 


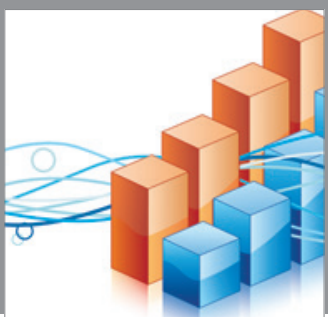

Advances in

Operations Research

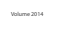

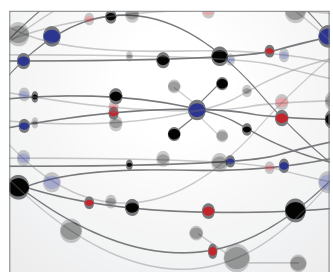

\section{The Scientific} World Journal
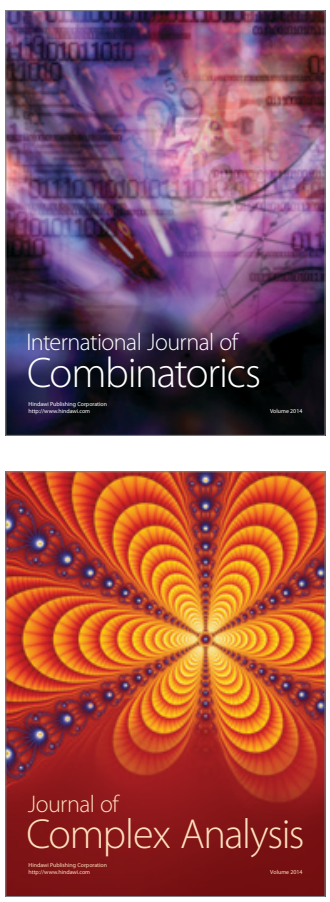

International Journal of

Mathematics and

Mathematical

Sciences
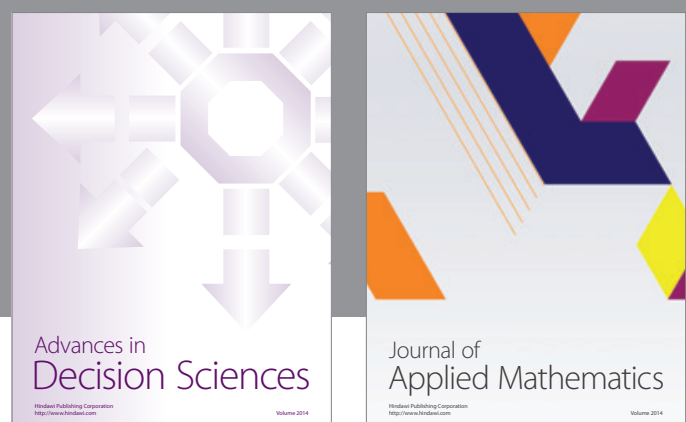

Journal of

Applied Mathematics
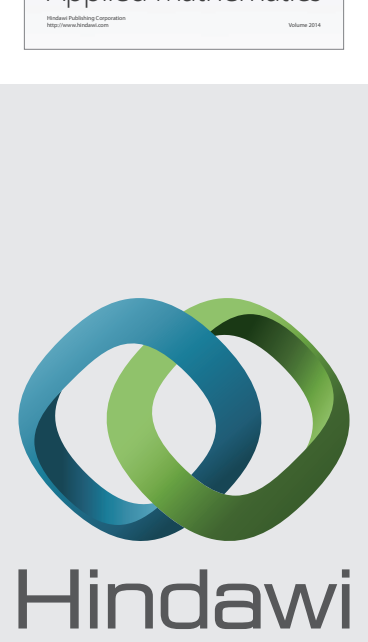

Submit your manuscripts at http://www.hindawi.com
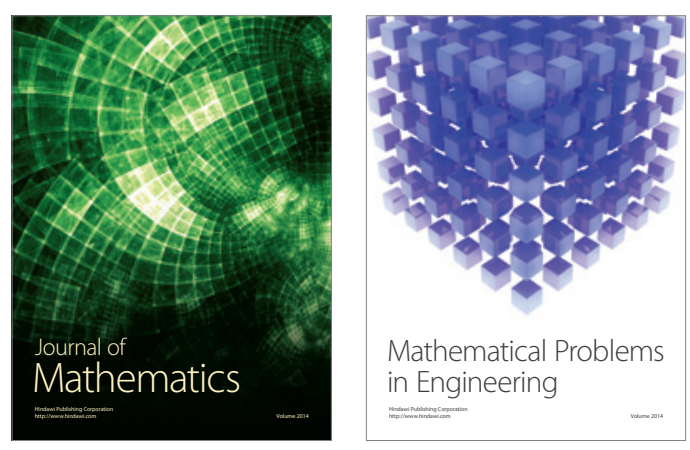

Mathematical Problems in Engineering
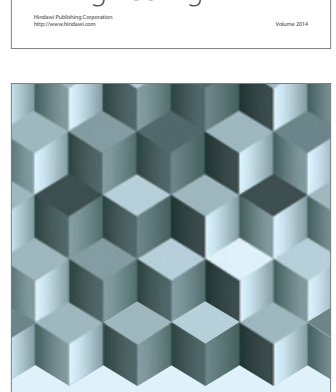

Journal of

Function Spaces
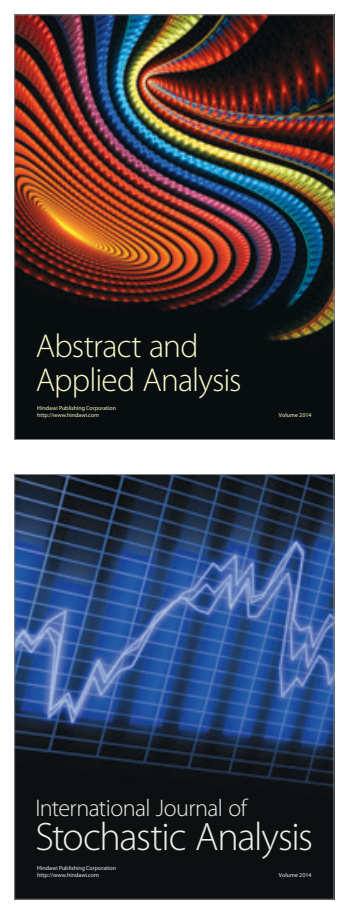

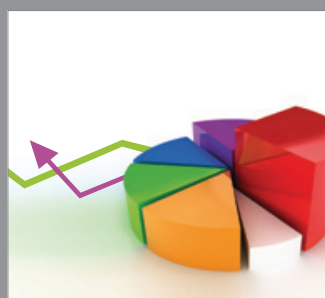

ournal of

Probability and Statistics

Promensencen
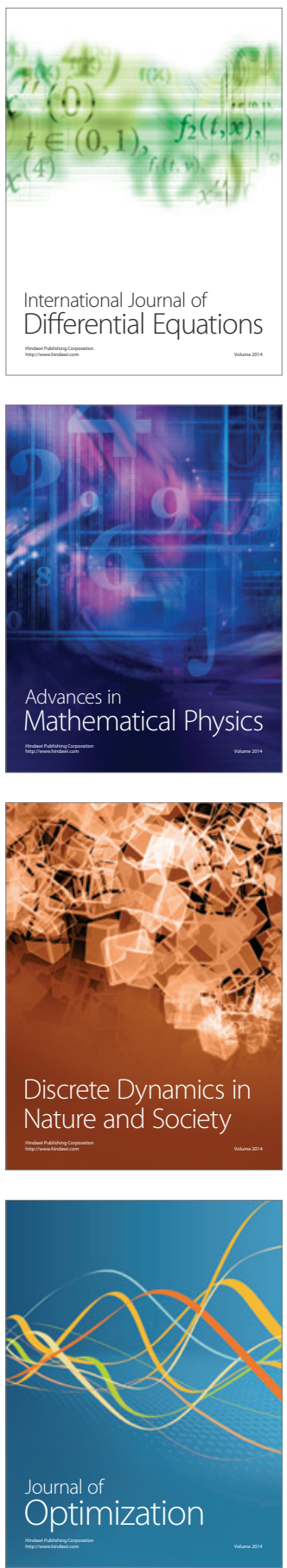